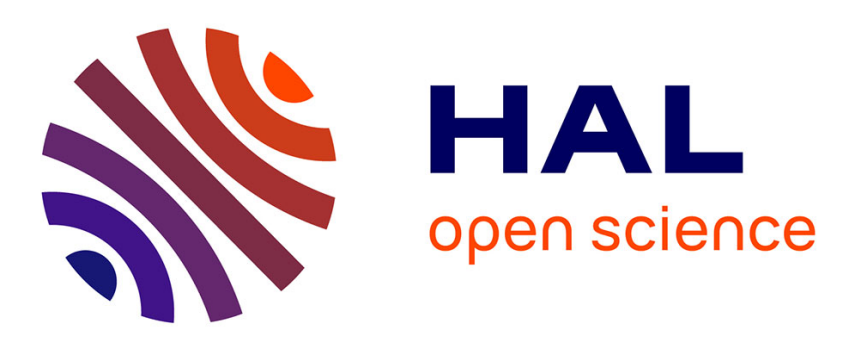

\title{
Computing minimal and maximal allowable transmission intervals for networked control systems using the hybrid systems approach
}

Stefan S.H.J. Heijmans, Romain Postoyan, Dragan Nesic, W.P. Maurice H. Heemels

\section{To cite this version:}

Stefan S.H.J. Heijmans, Romain Postoyan, Dragan Nesic, W.P. Maurice H. Heemels. Computing minimal and maximal allowable transmission intervals for networked control systems using the hybrid systems approach. IEEE Control Systems Letters, 2017, 1 (1), pp.56-61. 10.1109/LCSYS.2017.2702755 . hal-01528353

\section{HAL Id: hal-01528353 \\ https://hal.science/hal-01528353}

Submitted on 9 Mar 2021

HAL is a multi-disciplinary open access archive for the deposit and dissemination of scientific research documents, whether they are published or not. The documents may come from teaching and research institutions in France or abroad, or from public or private research centers.
L'archive ouverte pluridisciplinaire HAL, est destinée au dépôt et à la diffusion de documents scientifiques de niveau recherche, publiés ou non, émanant des établissements d'enseignement et de recherche français ou étrangers, des laboratoires publics ou privés. 


\title{
Computing Minimal and Maximal Allowable Transmission Intervals for Networked Control Systems Using the Hybrid Systems Approach
}

\author{
S.H.J. Heijmans, R. Postoyan, D. Nešić, Fellow, IEEE, and W.P.M.H. Heemels, Fellow, IEEE
}

\begin{abstract}
A popular design framework for networked control systems (NCSs) is the emulation-based approach combined with hybrid systems analysis techniques. In the rich literature regarding this framework, bounds on the maximally allowable transmission interval (MATI) are provided to guarantee stability properties of the NCS, while the minimal allowable transmission interval (MIATI) is always taken to be (essentially) zero. In this letter, we show for the first time how knowledge of the MIATI can also be exploited in the hybrid systems/emulation-based framework leading to (guaranteed) higher values for the MATI, while still obtaining stability of the NCS.
\end{abstract}

Index Terms-Networked control systems, stability of hybrid systems, Lyapunov methods.

\section{INTRODUCTION}

$\mathbf{N}$ ETWORKED control systems (NCSs) are systems in which the sensors, controllers, and actuators of the plant are physically distributed and communicate via (packet-based) digital channels. These systems have received considerable attention in recent years, motivated by the many benefits they offer with respect to conventional (wired) control systems, including greater flexibility, ease of maintenance, and low cost, weight and volume [1]. However, exploiting packet-based communication networks also comes with inevitable imperfections such as varying transmission intervals, delays, and

Manuscript received March 6, 2017; accepted April 20, 2017. Date of publication May 9, 2017; date of current version May 24, 2017. The work of S.H.J. Heijmans and W.P.M.H. Heemels is supported by the Innovational Research Incentives Scheme under the VIC grant "Wireless control systems: A new frontier in automation" (No. 11382) awarded by the Netherlands Organisation for Scientific Research (NWO) and Taskforce for Applied Research SIA. The work of R. Postoyan is partly supported by the ANR through COMPACS under Grant ANR-13-BS03-0004-02. The work of D. Nešić was supported by the Australian Research Council through the Discovery Project under Grant DP1094326. Recommended by Senior Editor J. Daafouz. (Corresponding author: S.H.J. Heijmans.)

S.H.J. Heijmans and W.P.M.H. Heemels are with the Department of Mechanical Engineering, Eindhoven University of Technology, 6006 GA Weert, The Netherlands (e-mail: s.h.j.heijmans@tue.nl)

R. Postoyan is with the Universite de Lorraine and the CNRS, 54516 Vandoeuvre-lès-Nancy, France.

D. Nešić is with the MIDAS Laboratory, Department of Electrical and Electronic Engineering, University of Melbourne, Parkville, VIC 3010, Australia. communication constraints that can all degrade the overall performance of the system and even lead to instability. Moreover, as the communication network is often shared by multiple sensor, controller, and actuator nodes, there is a need for so-called scheduling protocols that govern the access of these nodes to the network.

Several frameworks were developed in recent years to determine conditions on the network while still guaranteeing stability and performance properties. A popular two-step design approach herein is the so-called emulation-based method as advocated in [2] and [3] combined with hybrid systems analysis tools, reflected in the works [4]-[12]. The idea is to first design the controller for the plant while ignoring the communication constraints, i.e., ideal communication is assumed. In the second step, conditions on the network, e.g., bounds on transmission intervals and delays, are provided to guarantee closed-loop stability and performance.

Strikingly, in all of the above works [2]-[12] the minimal allowable transmission interval (MIATI) was always set to be (essentially) zero, although it is well-known that knowledge on the MIATI can lead to improved bounds on the maximal allowable transmission interval (MATI). Indeed, other frameworks available in the literature such as the timedelay approach or the discrete-time approach (with convex embeddings), see [13]-[15] or the recent survey [16] and the references therein, can incorporate strictly positive MIATIs and exploit this information to obtain less conservative estimates of the MATI. Given this 'gap' in the hybrid systems analysis framework for NCSs in the line of [4]-[12], it is the main objective in this letter to show how knowledge of positive lower bounds on the transmission interval (the MIATI) can be used in the emulation/hybrid-based NCS framework as in [4]-[6] leading to (guaranteed) higher values for the MATI, while obtaining stability of the NCS, see also [17] for a computational approach in this direction using sum of squares techniques. Here we focus on a more analytical approach in line with [2]-[12].

To this end, we consider the same fundamental NCS setup as in [3]-[6], which was extended in various directions later [7]-[12]. We show that, under essentially the same conditions as exploited in previous papers for the case of zero MIATI in, e.g., [4]-[6], we can provide a bound on the MATI that is guaranteed to be larger while still guaranteeing closed-loop stability. Instrumental in our analysis will be the concept of (minimal) dwell-time, see [18]-[20]. We believe that such new results providing larger 'stabilizing' values for 
the MATI by exploiting knowledge on the MIATI is useful as in practice, due to hardware limitations, transmissions of packets cannot occur infinitely fast and, therefore, a minimal amount of time between transmissions indeed always exists. We will illustrate the obtained improvements via a numerical example.

The remainder of this letter is organized as follows. After presenting the necessary notation, the considered class of systems is described in Section II including a suitable hybrid model for the NCS. In Section III, we present our main result, i.e., by exploiting knowledge on the MIATI we show that, based on the same stability conditions of [6], an improved bound on the MATI can be obtained. We also show in Section IV how the condition on the MIATI can be relaxed. In Section V, it is stated how the results can be reformulated in terms of LMIs when linear NCSs are considered. Finally, in Section VI our results are illustrated using an example, and in Section VII concluding remarks are given.

Notation: The set of real numbers is denoted by $\mathbb{R}$ and the sets of non-negative real numbers and integers by $\mathbb{R}_{\geq 0}$ and $\mathbb{N}$, respectively. For vectors $v_{1}, v_{2}, \ldots, v_{n} \in \mathbb{R}^{n}$, we denote by $\left(v_{1}, v_{2}, \ldots, v_{n}\right)$ the vector $\left[\begin{array}{llll}v_{1}^{\top} & v_{2}^{\top} & \ldots & v_{n}^{\top}\end{array}\right]^{\top}$, and by $|\cdot|$ and $\langle\cdot, \cdot\rangle$ the Euclidean norm and the usual inner product, respectively. We use the notation $r^{+}(t)=r\left(t^{+}\right)=\lim _{\tau \downarrow t} r(\tau)$, provided the limit exists. The $n$ by $n$ identity and zero matrices are denoted by $I_{n}$ and $0_{n}$, respectively. For a symmetric matrix $A \in \mathbb{R}^{n \times n}, \lambda_{\max }(A)$ denotes the largest eigenvalue of $A$.

\section{SYSTEM DESCRIPTION}

In this section, we introduce the NCS setup and a hybrid model describing the overall dynamics.

\section{A. Networked Control Configuration}

We consider the NCS as shown in Fig. 1, where the continuous-time plant $\mathcal{P}$ communicates with the controller $\mathcal{C}$ via the network $\mathcal{N}$. The plant and controller dynamics are given by

$$
\mathcal{P}:\left\{\begin{array}{l}
\dot{x}_{p}=f_{p}\left(x_{p}, \hat{u}\right) \\
y=g_{p}\left(x_{p}\right)
\end{array} \quad \text { and } \mathcal{C}:\left\{\begin{array}{l}
\dot{x}_{c}=f_{c}\left(x_{c}, \hat{y}\right) \\
u=g_{c}\left(x_{c}\right),
\end{array}\right.\right.
$$

where $x_{p} \in \mathbb{R}^{m_{x_{p}}}$ and $x_{c} \in \mathbb{R}^{m_{c}}$ denote the plant and controller state, $u \in \mathbb{R}^{m_{u}}$ the control input and $\hat{u} \in \mathbb{R}^{m_{u}}$ the most recently received control input by the plant, and $y \in \mathbb{R}^{m_{y}}$ the output and $\hat{y} \in \mathbb{R}^{m_{y}}$ the most recently received output of the plant. We assume that $f_{p}$ and $f_{c}$ are continuous, and $g_{p}$ and $g_{c}$ continuously differentiable.

To complete the description of the NCS setup, it has to be explained how the communication network $\mathcal{N}$ operates. This network $\mathcal{N}$ has a collection of sampling/transmission times $t_{j}$, $j \in \mathbb{N}$, which satisfy $0 \leq t_{0}<t_{1}<\ldots$. At a transmission time $t_{j}$, (parts of) the output $y$ and the input $u$ are sampled and transmitted over the network $\mathcal{N}$ to the controller $\mathcal{C}$ and the plant $\mathcal{P}$, respectively. In the considered setup, similar to [3]-[6], it is assumed that the transmission intervals are bounded by

$$
\tau_{\text {miati }} \leq t_{j+1}-t_{j} \leq \tau_{\text {mati }}, \quad j \in \mathbb{N},
$$

where $\tau_{\text {mati }}$ denotes the maximally allowable transmission interval (MATI) and $\tau_{\text {miati }}$ the minimal allowable transmission interval (MIATI), such that $0<\tau_{\text {miati }} \leq \tau_{\text {mati }}$.

In addition to this sequence of transmission times, the network $\mathcal{N}$ might also be subdivided in several (sensor and/or

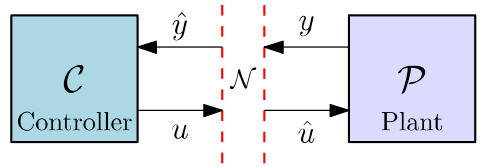

Fig. 1. The NCS setup as described in [2]-[6].

actuator) nodes, where each node corresponds to a subset of the entries $y / \hat{y}$ and/or $u / \hat{u}$. A scheduling protocol determines which of the nodes in the network is granted access to the network at a transmission time. After a node is granted access to the network, it collects and transmits the values of the corresponding entries in $y\left(t_{j}\right)$ and $u\left(t_{j}\right)$, which results in an update according to

$$
\begin{aligned}
& \hat{y}\left(t_{j}^{+}\right)=y\left(t_{j}\right)+h_{y}\left(j, e\left(t_{j}\right)\right) \\
& \hat{u}\left(t_{j}^{+}\right)=u\left(t_{j}\right)+h_{u}\left(j, e\left(t_{j}\right)\right),
\end{aligned}
$$

where the function $h:=\left(h_{y}, h_{u}\right)$ with $h: \mathbb{N} \times \mathbb{R}^{m_{e}} \rightarrow \mathbb{R}^{m_{e}}$ models the scheduling protocol, see [5]-[7], [9], and where $e$ denotes the network-induced error defined by

$$
e:=\left[\begin{array}{l}
e_{y} \\
e_{u}
\end{array}\right]=\left[\begin{array}{l}
\hat{y}-y \\
\hat{u}-u
\end{array}\right] \text {. }
$$

Finally, it is assumed that $\hat{y}$ and $\hat{u}$ are constant in between two successive transmissions (zero-order-hold $(\mathrm{ZOH})$ ). However, this can easily be modified, if desired, see [5].

Observe now that, strikingly, in [2]-[12] the lower bound $\tau_{\text {miati }}$ was always allowed (and often even assumed) to be arbitrarily small (and thus essentially zero) as it was only imposed to prevent Zeno behavior. Clearly, due to hardware limitations in reality such a positive lower bound $\tau_{\text {miati }}$ on the transmission intervals always exists and can be exploited to obtain a larger bound on the MATI, see Section III below.

Remark 1: Similar to [5]-[7], for simplicity of exposition, we only focus on the control configuration as in Fig. 1. However, the results presented in this letter also apply to other configurations such as the case of static state feedback control and the case where either $\hat{u}=u$ or $\hat{y}=y$, meaning that the corresponding signals are not transmitted over a (shared) network, but are continuously available. To illustrate this flexibility, a numerical example in which $\hat{u}=u$ is considered in Section VI.

\section{B. A Hybrid Modeling Framework}

The above NCS setup can be rewritten in the hybrid system formalism advocated in [5]. To do so, similar to [5]-[7], we introduce the timer $\tau \in \mathbb{R}_{\geq 0}$, which keeps track of the time elapsed since the last transmission and resets to zero after a transmission has occurred, and the counter $\kappa \in \mathbb{N}$, which keeps track of the number of transmissions. Using these auxiliary variables, the NCS can be expressed as the hybrid model ${ }^{1}$

$$
\mathcal{H}:\left\{\begin{array}{lll}
\dot{\xi}=F(\xi) & \text { when } \tau \in\left[0, \tau_{\text {mati }}\right] \\
\xi^{+}=G(\xi) & \text { when } \tau \in\left[\tau_{\text {miati }}, \infty\right)
\end{array}\right.
$$

with the full state of the hybrid system

$$
\xi:=\left(\left(x_{p}, x_{c}\right), e, \tau, \kappa\right) \in \mathbb{X}:=\mathbb{R}^{m_{x}} \times \mathbb{R}^{m_{e}} \times \mathbb{R}_{\geq 0} \times \mathbb{N}
$$

\footnotetext{
${ }^{1}$ For details and terminology on hybrid systems of the form (4), see [21]
} 
and where $F(\xi):=(f(x, e), g(x, e), 1,0)$ and $G(\xi):=(x$, $h(\kappa, e), 0, \kappa+1)$ with $x:=\left(x_{p}, x_{c}\right) \in \mathbb{R}^{m_{x}}$, and

$$
\begin{aligned}
f(x, e) & =\left[\begin{array}{l}
f_{p}\left(x_{p}, g_{c}\left(x_{c}\right)+e_{u}\right) \\
f_{c}\left(x_{c}, g_{p}\left(x_{p}\right)+e_{y}\right)
\end{array}\right] \\
g(x, e) & =\left[\begin{array}{l}
-\frac{\partial g_{p}}{\partial x_{p}} f_{p}\left(x_{p}, g_{c}\left(x_{c}\right)+e_{u}\right) \\
-\frac{\partial g_{c}}{\partial x_{c}} f_{c}\left(x_{c}, g_{p}\left(x_{p}\right)+e_{y}\right)
\end{array}\right] .
\end{aligned}
$$

We are interested in the stability of this hybrid model (4).

Definition 1: For the system $\mathcal{H}$ given by (4), the set

$$
\mathcal{E}=\{\xi=(x, e, \tau, \kappa) \in \mathbb{X} \mid x=0 \wedge e=0\}
$$

is said to be uniformly globally exponentially stable (UGES) if there exists a function $\beta: \mathbb{R}_{\geq 0} \times \mathbb{R}_{\geq 0} \rightarrow \mathbb{R}_{\geq 0}$ of the form $\beta(r, s)=K r \exp (-c s)$ for some $K, c>0$ such that for any initial condition $\xi(0,0) \in \mathbb{X}$, all corresponding maximal solutions $\xi$ satisfy for all $(t, j) \in \operatorname{dom} \xi$

$$
|(x(t, j), e(t, j))| \leq \beta(|(x(0,0), e(0,0))|, t+j) .
$$

\section{Main REsult}

In [6], a stability result has been presented for NCSs based on Lyapunov-arguments for hybrid systems. In particular, the proof was based on the construction of a Lyapunov function that strictly decreases during flow of the hybrid system (4) (i.e., in between transmission times) and does not increase at a jump of the hybrid system (4) (i.e., when an update of the networked values occurs). As a result, conditions were obtained on the MATI such that stability is guaranteed. However, as we know that the hybrid system (4) 'dwells' for at least $\tau_{\text {miati }}>0$ time units (and, hence, based on the conditions presented in [6] the Lyapunov function decreases during this time), we observe that the strict non-increase of the Lyapunov function at jumps can be relaxed, leading to the following result.

Theorem 1: Consider the hybrid system $\mathcal{H}$ as in (4). Assume there exist a function $W: \mathbb{N} \times \mathbb{R}^{m_{e}} \rightarrow \mathbb{R}_{\geq 0}$ that is locally Lipschitz in its second argument, a locally Lipschitz function $V: \mathbb{R}^{m_{x}} \rightarrow \mathbb{R}_{\geq 0}$, a continuous function $H: \mathbb{R}^{m_{x}} \rightarrow \mathbb{R}$, and the constants $\lambda \in(0,1), L \geq 0, \underline{\alpha}_{W}, \bar{\alpha}_{W}, \underline{\alpha}_{V}, \bar{\alpha}_{V}>0$ and $0<\varepsilon<\gamma$ such that the following hold:

1) For all $\kappa \in \mathbb{N}$ and $e \in \mathbb{R}^{m_{e}}$

$$
\begin{aligned}
\underline{\alpha}_{W}|e| \leq W(\kappa, e) & \leq \bar{\alpha}_{W}|e| \\
W(\kappa+1, h(\kappa, e)) & \leq \lambda W(\kappa, e) .
\end{aligned}
$$

2) For all $\kappa \in \mathbb{N}, x \in \mathbb{R}^{m_{x}}$, and almost all $e \in \mathbb{R}^{m_{e}}$

$$
\left\langle\frac{\partial W(\kappa, e)}{\partial e}, g(x, e)\right\rangle \leq L W(\kappa, e)+H(x) .
$$

3) For all $x \in \mathbb{R}^{m_{x}}$

$$
\underline{\alpha}_{V}|x|^{2} \leq V(x) \leq \bar{\alpha}_{V}|x|^{2} .
$$

4) For all $e \in \mathbb{R}^{m_{e}}$, and almost all $x \in \mathbb{R}^{m_{x}}$

$$
\begin{aligned}
& \langle\nabla V(x), f(x, e)\rangle \\
& \quad \leq-\varepsilon^{2}|x|^{2}+\underline{\alpha}_{W}^{2}\left(\gamma^{2}-\varepsilon^{2}\right)|e|^{2}-H^{2}(x) .
\end{aligned}
$$

5) For a given $\tau_{\text {miati }}>0, \tau_{\text {mati }}$ satisfies the bound

$$
\tau_{\text {mati }} \leq \begin{cases}\frac{1}{L r} \arctan \left(\frac{r(1-\sigma)}{2 \frac{\sigma}{1+\sigma}\left(\frac{\gamma}{L}-1\right)+1+\sigma}\right) & \gamma>L \\ \frac{1}{L} \frac{1-\sigma}{1+\sigma} & \gamma=L \\ \frac{1}{L r} \operatorname{arctanh}\left(\frac{r(1-\sigma)}{2 \frac{\sigma}{1+\sigma}\left(\frac{\gamma}{L}-1\right)+1+\sigma}\right) & \gamma<L \\ \frac{1}{\gamma} \arctan \left(\frac{(1+\sigma)(1-\sigma)}{2 \sigma}\right) & L=0\end{cases}
$$

with $r=\sqrt{\left|\left(\frac{\gamma}{L}\right)^{2}-1\right|}$ for some constant $\sigma>\lambda \Lambda$ where

$$
\Lambda=\max \left\{e^{-\tilde{\varepsilon} \bar{\alpha}_{V}^{-1} \tau_{\text {miati }}}, e^{-W_{L}\left(\tilde{\varepsilon}\left(\gamma \lambda^{-1} \bar{\alpha}_{W}^{2}\right)^{-1} \tau_{\text {miati }}\right)}\right\},
$$

$\tilde{\varepsilon}:=\frac{1}{2} \varepsilon^{2} \min \left\{1, \underline{\alpha}_{W}^{2}\right\}$, and where $W_{L}$ is the so-called Lambert $W$-function, which is implicitly defined as the function satisfying $W_{L}(z) e^{W_{L}(z)}=z$, see [22].

Then, the set $\mathcal{E}$ in (6) is UGES.

The proof is given in Appendix A. In practice, we will take $\sigma$ as close as possible to its lower bound since this results in the highest value for $\tau_{\text {mati }}$. Observe now that for any $\tau_{\text {miati }}>0$, it holds that $\Lambda<1$, and, hence, we can always take $\sigma=\lambda$, in which case we recover the MATI bound of [6, Th. 1]. This observation also implies that, for any positive $\tau_{\text {miati }}$, there exists a $\sigma<\lambda$. As a result, under essentially the same conditions as presented in [6] (i.e., (7)-(10) do not change for any $\sigma)$, we can always obtain a higher bound on $\tau_{\text {mati }}$ than can be obtained using the result from [6] (for which essentially $\tau_{\text {miati }}=0$ and $\sigma=\lambda$ ). Hence, exploiting a positive MIATI is guaranteed to improve the MATI.

However, it should also be noted that the value of $\Lambda$ strongly depends on the values of $\varepsilon$ and

$$
\bar{\alpha}_{U}=\max \left\{\bar{\alpha}_{V}, \gamma \sqrt{\mu} \lambda^{-1} \bar{\alpha}_{W}^{2}\right\}
$$

which are strongly related to the convergence rate of the system to the equilibrium set $\mathcal{E}$. In particular, UGES of the set $\mathcal{E}$ can already be established for a small value of $\varepsilon$ (although at the cost of a slow convergence rate to $\mathcal{E}$, see $(25)$ in the proof). Hence, as often $\bar{\alpha}_{U}>1$, such a choice for $\varepsilon$ would imply that the value of $\sigma$ should be chosen very close to $\lambda$ (as the value of $\Lambda$ is very close to 1 ), making the improvement of $\tau_{\text {mati }}$ with respect to the results of [6] only marginal. On the other hand, when the convergence rate of the system is important and, hence, the value of $\varepsilon$ is taken relatively large, the improvement of $\tau_{\text {mati }}$ with respect to the results of [6] can be quite substantial, as will be shown in the numerical example in Section VI. Moreover, in the following section we will show that the condition on $\sigma$ can be relaxed.

Remark 2: All the conditions in Theorem 1 are checkable and natural in the context of NCSs, see also [2]-[6]. In particular, (7) corresponds to the existence of a so-called UGES scheduling protocol, a notion introduced in [5], and is therefore related to the error dynamics of the NCS at jumps. As shown in [5], various protocols exist which satisfy the conditions (7), including the round-robin (RR) and try-once-discard (TOD) protocols. Indeed, (7a) is satisfied for $\underline{\alpha}_{W, R R}=\underline{\alpha}_{W, T O D}=1, \bar{\alpha}_{W, R R}=\sqrt{\ell}$, and $\bar{\alpha}_{W, T O D}=1$ and (7b) for $\lambda_{T O D}=\lambda_{R R}=\sqrt{(\ell-1) / \ell}$ with $\ell$ the number of nodes in the network. Moreover, based on the discussion in [5, Remark 11], it follows that (8) is also not hard to verify for a large class of systems. This is, for instance, illustrated in Section $\mathrm{V}$ for the linear case. Conditions (9) and (10) are related to finding a Lyapunov function that guarantees a robust stability property for the closed-loop (state) dynamics. The remaining part is then to connect (7) and (8) on one hand and (9) and (10) on the other hand, which is done via (11) by guaranteeing that for sufficiently fast transmissions a small gain condition holds.

\section{RELAXING THE MIATI}

As mentioned above, the dependence of $\tau_{\text {miati }}$ on $\bar{\alpha}_{U}$ limits the value that $\Lambda$ (cf. $\sigma$ ) can attain, and, thereby, the improvement we can obtain for the bound on $\tau_{\text {mati }}$. Therefore, 
in this section, we propose a slightly modified version of Theorem 1, which in some cases, for instance, for the numerical example in Section VI, will further improve the bound on $\tau_{\text {mati }}$.

Theorem 2: Consider the hybrid system $\mathcal{H}$ as in (4). Assume there exist a function $W: \mathbb{N} \times \mathbb{R}^{m_{e}} \rightarrow \mathbb{R}_{>0}$ that is locally Lipschitz in its second argument, a locally Lipschitz function $V: \mathbb{R}^{m_{x}} \rightarrow \mathbb{R}_{>0}$, a continuous function $H: \mathbb{R}^{m_{x}} \rightarrow \mathbb{R}$, and the constants $\lambda \in(0,1), L \geq 0, \underline{\alpha}_{W}, \bar{\alpha}_{W}, \underline{\alpha}_{V}, \bar{\alpha}_{V}>0$ and $0<\varepsilon<\gamma$ such that the following holds:

1) For all $\kappa \in \mathbb{N}, e \in \mathbb{R}^{m_{e}}$, and $x \in \mathbb{R}^{m_{x}}$ (7) and (9) hold.

2) For all $\kappa \in \mathbb{N}, x \in \mathbb{R}^{m_{x}}$, and almost all $e \in \mathbb{R}^{m_{e}}$

$$
\left\langle\frac{\partial W(\kappa, e)}{\partial e}, g(x, e)\right\rangle \leq\left(L-\frac{1}{2} \varepsilon\right) W(\kappa, e)+H(x) .
$$

3) For all $\kappa \in \mathbb{N}, e \in \mathbb{R}^{m_{e}}$, and almost all $x \in \mathbb{R}^{m_{x}}$

$$
\langle\nabla V(x), f(x, e)\rangle \leq-\varepsilon^{2} V(x)+\gamma^{2} W^{2}(\kappa, e)-H^{2}(x) .
$$

4) For a given $\tau_{\text {miati }}>0, \tau_{\text {mati }}$ satisfies the bound (11) for some constant $\sigma>\lambda \Lambda$ with $\Lambda=e^{-\frac{1}{2} \varepsilon^{2} \tau_{\text {miati }} \text {. }}$

Then, the set $\mathcal{E}$ in (6) is UGES.

The proof is given in Appendix B. Note that when (14) holds, also (8) is satisfied and that when (10) holds, also (15) is satisfied. Moreover, for a sufficiently small value of $\varepsilon$ the reverse is also implied, making Theorems 1 and 2 equivalent to each other. However, as can be seen from item 4) in Theorem $2, \Lambda$ solely depends on the value of $\varepsilon$ and not on the value of $\bar{\alpha}_{U}$ as was the case in Theorem 1. As a result, for the same value of $\varepsilon$ and $\tau_{\text {miati }}$ in Theorems 1 and 2 , the value of $\sigma$ can be taken smaller in the case of Theorem 2. As a result for small values of $\varepsilon$ we in general obtain better results using Theorem 2 than using Theorem 1, as will also be shown in the numerical example of Section VI.

\section{The LINEAR CASE}

In this section, we discuss how to systematically construct the functions and constants satisfying the conditions presented in Section III in the case that the NCS is composed of a linear plant and a linear controller. Consider hereto NCSs for which $f(x, e)$ and $g(x, e)$ in (5) can be reduced to the form of

$$
f(x, e)=\mathbf{A} x+\mathbf{E} e \text { and } g(x, e)=\mathbf{C} x+\mathbf{F} e .
$$

As shown before in, for instance, [11], to systematically verify the conditions of Theorem 1 it is possible to reformulate the presented conditions into LMI-based conditions. Consider hereto condition (8) and assume now that for almost all $e \in \mathbb{R}^{m_{e}}$ and all $\kappa \in \mathbb{N}$ it holds that

$$
\left|\frac{\partial W(\kappa, e)}{\partial e}\right| \leq M
$$

for some constant $M>0$. This condition can be directly used to conclude that the function $W$ is locally Lipschitz. For all the considered protocols in [2]-[6] such a constant exists. More precisely, for the RR, and TOD protocols it holds that condition (17) is fulfilled for $M_{R R}=\sqrt{\ell}$ and $M_{T O D}=1$, respectively, as shown in [9]. Moreover, when using (16), it follows for the error dynamic that

$$
|\dot{e}| \leq|\mathbf{C} x|+|\mathbf{F} e|
$$

and hence, in (8), $L=M \underline{\alpha}_{W}^{-1}|\mathbf{F}|$ and $H(x)=M|\mathbf{C} x|$. Using this result in (10), it can be directly obtained that for the right-hand side it holds that

$$
\begin{aligned}
& -\varepsilon^{2}|x|^{2}+\underline{\alpha}_{W}^{2}\left(\gamma^{2}-\varepsilon^{2}\right)|e|^{2}-H^{2}(x)=\left[\begin{array}{l}
x \\
e
\end{array}\right]^{\top} J\left[\begin{array}{l}
x \\
e
\end{array}\right] \\
& \text { with } J:=\left[\begin{array}{cc}
-\varepsilon^{2} I_{m_{x}}-M^{2} \mathbf{C}^{\top} \mathbf{C} & 0 \\
0 & \underline{\alpha}_{W}^{2}\left(\gamma^{2}-\varepsilon^{2}\right) I_{m_{e}}
\end{array}\right] .
\end{aligned}
$$

To arrive now at an LMI-based condition, which guarantees UGES, also the left-hand side of (10) needs to be evaluated. Therefore, we take $V(x)=x^{\top} X_{\mathbf{T}} x$ with $X_{\mathbf{T}}$ being a symmetric positive definite matrix of size $m_{x} \times m_{x}$ such that (9) is satisfied by definition. We obtain now that

$$
\begin{aligned}
& \langle\nabla V(x), \mathbf{A} x+\mathbf{E} e\rangle \\
& \quad=x^{\top}\left(\mathbf{A}^{\top} X_{\mathbf{T}}+X_{\mathbf{T}} \mathbf{A}\right) x+x^{\top} X_{\mathbf{T}} \mathbf{E} e+e^{\top} \mathbf{E}^{\top} X_{\mathbf{T}} x .
\end{aligned}
$$

Theorem 1 can now be rewritten using the following LMIbased conditions.

Theorem 3: Consider the hybrid system $\mathcal{H}$ as in (4) with (16). Assume there exist a function $W: \mathbb{N} \times \mathbb{R}^{m_{e}} \rightarrow \mathbb{R}_{\geq 0}$, a symmetric positive definite matrix $X_{\mathbf{T}}$, and strictly positive real numbers $M, \underline{\alpha}_{W}, \bar{\alpha}_{W}, \lambda \in[0,1)$, and $0<\varepsilon<\gamma$ such that the following holds:

1) For all $\kappa \in \mathbb{N}$ and $e \in \mathbb{R}^{m_{e}}$ (7) holds.

2) For all $\kappa \in \mathbb{N}$, and for almost all $e \in \mathbb{R}^{m_{e}}$ (17) holds.

3) $\left[\begin{array}{cc}\mathbf{A}^{\top} X_{\mathbf{T}}+X_{\mathbf{T}} \mathbf{A}+\varepsilon^{2} I_{m_{x}}+M^{2} \mathbf{C}^{\top} \mathbf{C} & X_{\mathbf{T}} \mathbf{E} \\ \mathbf{E}^{\top} X_{\mathbf{T}} & -\underline{\alpha}_{W}^{2}\left[\gamma^{2}-\varepsilon^{2}\right] I_{m_{e}}\end{array}\right] \preceq 0$.

4) For a given $\tau_{\text {miati }}>0$, $\tau_{\text {mati }}$ satisfies the bound (11) with $L=M \underline{\alpha}_{W}^{-1}|\mathbf{F}|$ and (12) where $\bar{\alpha}_{V}=\lambda_{\max }\left(X_{\mathbf{T}}\right)$.

Then, the set $\mathcal{E}$ in (6) is UGES.

Remark 3: In a similar fashion, a LMI condition for Theorem 2 can be obtained. In particular, in Theorem 3 we would then have that item 3 ) is given by

$$
\left[\begin{array}{cc}
\mathbf{A}^{\top} X_{\mathbf{T}}+X_{\mathbf{T}} \mathbf{A}+\varepsilon^{2} X_{\mathbf{T}}+M^{2} \mathbf{C}^{\top} \mathbf{C} & X_{\mathbf{T}} \mathbf{E} \\
\mathbf{E}^{\top} X_{\mathbf{T}} & -\underline{\alpha}_{W}^{2} \gamma^{2} I_{m_{e}}
\end{array}\right] \preceq 0
$$

and that in item 4) $L=M \underline{\alpha}_{W}^{-1}|\mathbf{F}|+\frac{1}{2} \varepsilon$ and $\Lambda=e^{-\frac{1}{2} \varepsilon^{2} \tau_{\text {miati }}}$.

\section{NUMERICAL EXAMPLE}

To illustrate the application of our results, we consider the example of stabilizing an open-loop unstable plant $\mathcal{P}$ with an state-feedback controller $\mathcal{C}$ given by

$$
\mathcal{P}: \dot{x}_{p}=A_{\mathbf{P}} x_{p}+B_{\mathbf{P}} u \text { and } \mathcal{C}: u=-K \hat{x}
$$

with $A_{\mathbf{P}}=\frac{1}{5}\left(\begin{array}{ll}-4 & 1 \\ -2 & 3\end{array}\right), \quad B_{\mathbf{P}}=\left(\begin{array}{c}-1 \\ 2\end{array}\right)$, and $K=\left(\begin{array}{ll}-0.2 & 0.5\end{array}\right)$. In this case we assume that only the states are transmitted over the network, i.e., we have for the error dynamics that $e=\hat{x}-x$ with the number of nodes in the network $\ell=2$, see also Remark 1. As a result, we have that the closed-loop dynamics are given by (16) with

$$
\begin{array}{ll}
\mathbf{A}:=A_{\mathbf{p}}-B_{\mathbf{P}} K C_{\mathbf{P}}, & \mathbf{E}:=-B_{\mathbf{P}} K \\
\mathbf{C}:=-C_{\mathbf{P}} \mathbf{A}, & \mathbf{F}:=-C_{\mathbf{P}} \mathbf{E}
\end{array}
$$

Using the various theorems, a bound on $\tau_{\text {mati }}$ can be computed for the system (19) with the TOD protocol and this bound can be compared to bound that can be obtained using the results of [6] (cf. $\mu=1$ in Theorem 1/3). The results for $\varepsilon=1$, $\varepsilon=0.8, \varepsilon=0.5$, and $\varepsilon=0.1$ can be seen in Table I. Here $\tau_{\text {mati }}^{\text {old }}$ represents the value obtained using the results from [6], $\tau_{\text {mati }}^{\text {new }}$ the value computed using Theorem 3 , and $\tau_{\text {mati }}^{n e w^{+}}$the value computed using the LMI version of Theorem 2, see Remark 3, 
TABLE I

THE BOUND ON $\tau_{\text {mati }}$ COMPUTED FOR VARIOUS VALUES OF $\varepsilon$

\begin{tabular}{|c|c|c|c|c|}
\hline$\varepsilon$ & $\tau_{\text {mati }}^{\text {old }}(\sigma=\lambda)$ & $\tau_{\text {mati }}^{\text {new }}$ & $\tau_{\text {mati }}^{\text {new }}$ & Improvement \\
\hline 1 & 0.0743 & 0.0748 & - & $0.68 \%$ \\
0.8 & 0.0852 & 0.0861 & 0.0441 & $1.04 \%$ \\
0.5 & 0.1071 & 0.1081 & 0.1222 & $14.07 \%$ \\
0.1 & 0.1399 & 0.1401 & 0.1414 & $1.04 \%$ \\
\hline
\end{tabular}

where we took the value of $\tau_{\text {miati }}$ equal to $\tau_{\text {mati }}^{\text {old }}$ or as high as possible in the cases that $\tau_{\text {mati }}^{n e w^{+}}<\tau_{\text {mati }}^{\text {old }}$.

As argued at the end of Section III, $\tau_{\text {mati }}^{\text {new }}$ is always larger than $\tau_{\text {mati }}^{\text {old }}$, a conclusion that is illustrated by the results in Table I. Moreover, while it cannot be guaranteed that $\tau_{\text {mati }}^{n e w^{+}}$ is always larger than $\tau_{m a t i}$ (or can even be computed), for small $\varepsilon$ it follows that $\tau_{\text {mati }}^{\text {new }}$ indeed can result in a further improvement of $\tau_{\text {mati }}^{\text {old }}$

On the other hand, it should also be noted that we in general obtain the largest improvement on the bound for the MATI for higher values of $\varepsilon$ as, logically, we can in this case take $\sigma$ small. Unfortunately however, $\tau_{\text {mati }}$ itself is in this case often smaller than what we can achieve with smaller values for $\varepsilon$ (although the convergence to the equilibrium set $\mathcal{E}$ is faster) Therefore, improving even further upon the results for small values of $\varepsilon$ is a topic of future research.

\section{CONCLUSION}

In this letter, we have provided an extension to the stability analysis of NCS based on the emulation/hybrid systems approach. In particular, it was shown that under the same conditions as in [6], a guaranteed improvement for the MATI can be realized, while ensuring UGES for the NCS by exploiting the fact that, due to hardware limitations, there exists a so-called positive minimal allowable transmission interval (MIATI). We foresee that the improvements and insights presented in this letter can possible inspire even sharper analysis tools.

\section{APPENDIX A}

PROOF OF THEOREM 1

The proof is based on Lyapunov-based arguments for hybrid systems [21, Proposition 3.29], which are adapted to allow for a relaxation based on dwell-time conditions, see [19], [20]. In particular, the proof is based on the construction of a (Lyapunov) function $U: \mathbb{R}^{m_{x}} \times \mathbb{R}^{m_{e}} \times \mathbb{R}_{\geq 0} \times \mathbb{N}$ that is locally Lipschitz in its first two arguments and the existence of constants $\lambda_{0}, \underline{\alpha}_{U}, \bar{\alpha}_{U}>0$, and $\mu \geq 1$ such that for (almost) all $\xi \in \mathbb{X}$

$$
\begin{array}{rlrl}
\underline{\alpha}_{U}\left|\xi_{c}\right|^{2} \leq U(\xi) & \leq \bar{\alpha}_{U}\left|\xi_{c}\right|^{2} & \\
U(G(\xi))-\mu U(\xi) & \leq 0, & \text { when } \tau \in\left[\tau_{\text {miati }}, \infty\right) \\
\langle\nabla U(\xi), F(\xi)\rangle & \leq-\lambda_{0} U(\xi) \text { when } \tau \in\left[0, \tau_{\text {mati }}\right]
\end{array}
$$

with $F(\xi)$ and $G(\xi)$ as in (4) and $\xi_{c}:=(x, e)$.

To prove that the conditions of (20) indeed are sufficient for the set $\mathcal{E}$ in (6) to be UGES, we aim to derive from $U$ a Lyapunov function $\mathcal{U}$ that satisfies the Lyapunov conditions for hybrid systems as stated in the proof of [6, Th. 1]. Consider to this end, for some $\tilde{\lambda} \in\left(0, \lambda_{0}\right)$ the function, $\xi \in \mathbb{X}$,

$$
\mathcal{U}(\xi)=e^{\tilde{\lambda} \tau} U(\xi)
$$

which is locally Lipschitz in its first two arguments. Recall that $\tau \in \mathbb{R}_{\geq 0}$ models the timer, i.e., $\dot{\tau}=1$ and $\tau^{+}=0$. As such, we observe that (20c) implies during flows with $\tau \in\left[0, \tau_{\text {mati }}\right]$ that

$$
\begin{aligned}
\langle\nabla \mathcal{U}(\xi), F(\xi)\rangle & =\tilde{\lambda} \dot{\tau} e^{\tilde{\lambda} \tau} U(\xi)+e^{\tilde{\lambda} \tau}\langle\nabla U(\xi), F(\xi)\rangle \\
& \stackrel{(20 \mathrm{c})}{\leq}\left(\tilde{\lambda}-\lambda_{0}\right) e^{\tilde{\lambda} \tau} U(\xi) .
\end{aligned}
$$

Hence, we indeed have that the function $\mathcal{U}$ is strictly decreasing between jump times since $\tilde{\lambda}<\lambda_{0}$. At a jump of the system, as mentioned above, $\tau$ is reset to zero, which, in combination with (20b), implies that for $\xi \in \mathbb{X}$ with $\tau \geq \tau_{\text {miati }}$

$$
\mathcal{U}(G(\xi))=e^{\tilde{\lambda} \cdot 0} U(G(\xi)) \leq \mu U(\xi)=\mu e^{-\tilde{\lambda} \tau} \mathcal{U}(\xi) .
$$

Since we know that two consecutive jumps of the system are separated by at least $\tau_{\text {miati }}$ time units, we obtain that when

$$
\mu e^{-\tilde{\lambda} \tau} \leq 1, \tau \geq \tau_{\text {miati }} \Leftrightarrow \mu \leq e^{\tilde{\lambda} \tau_{\text {miati }}}
$$

is satisfied, we have that (22) results in

$$
\mathcal{U}(G(\xi)) \leq \mu e^{-\tilde{\lambda} \tau} \mathcal{U}(\xi) \leq e^{\tilde{\lambda} \tau_{\text {miati }}} e^{-\tilde{\lambda} \tau_{\text {miati }}} \mathcal{U}(\xi) \leq \mathcal{U}(\xi)
$$

Note that there always exists a value for $\mu$ sufficiently close to 1 that satisfies (23) as $\tau_{\text {miati }}>0$. At the end of the proof we will show how, based on $\tau_{\text {miati }}$ and the conditions in Theorem 1, the 'optimal' value for $\mu \geq 1$ can be obtained. From this result we can conclude that indeed the conditions of (20) are sufficient to guarantee UGES of the set $\mathcal{E}$ in (6) by following similar lines as in the proof of $[6$, Th. 1].

To show now that the hypotheses of Theorem 1 indeed can be used to obtain a Lyapunov function $U$ such that the conditions in (20) with (23) are satisfied, we consider the function $\phi:\left[0, \tau_{\text {mati }}\right] \times \mathbb{Z} \rightarrow \mathbb{R}_{\geq 0}$, which evolves according to

$$
\frac{\mathrm{d}}{\mathrm{d} \tau} \phi(\tau)=-2 L \phi(\tau)-\gamma\left(\phi^{2}(\tau)+1\right)
$$

for $\tau \in\left[0, \tau_{\text {mati }}\right]$. In [6], it has been shown that choosing the initial condition to be $\phi(0)=\sigma^{-1}$ for some constant $\sigma \leq 1$, the function $\phi$ satisfies $\phi\left(\tau_{\text {mati }}\right)=\sigma$ and $\phi(\tau) \in\left[\sigma, \sigma^{-1}\right]$ for $\tau \in\left[0, \tau_{\text {mati }}\right]$. Using this function $\phi$, we define the candidate Lyapunov function, see also [6], as, for $\xi \in \mathbb{X}$,

$$
U(\xi)=V(x)+\gamma \phi(\tau) W^{2}(\kappa, e) .
$$

Since $\phi(\tau) \geq \sigma>0$ for all $\tau \in\left[0, \tau_{\text {mati }}\right]$ and as we have that (7a) and (9) hold, it can be concluded that (20a) is satisfied for

$$
\underline{\alpha}_{U}=\min \left\{\underline{\alpha}_{V}, \gamma \sigma \underline{\alpha}_{W}^{2}\right\} \text { and } \bar{\alpha}_{U}=\max \left\{\bar{\alpha}_{V}, \gamma \sigma^{-1} \bar{\alpha}_{W}^{2}\right\} \text {. }
$$

To prove that also (20b) holds under the conditions of Theorem 1, consider the situation in which an update of the networked values is performed, i.e., a jump occurs in the hybrid system (4). This gives, for $\xi \in \mathbb{X}$ with $\tau \geq \tau_{\text {mati }}$,

$$
\begin{aligned}
U(G(\xi)) & =V(x)+\gamma \phi(0) W^{2}(\kappa+1, h(\kappa, e)) \\
& \stackrel{(7 \mathrm{~b})}{\leq} V(x)+\gamma \sigma^{-1} \lambda^{2} W^{2}(\kappa, e) \leq \mu U(\xi)
\end{aligned}
$$

when $\sigma=\frac{\lambda}{\sqrt{\mu}}$ and, hence, (20b) indeed holds.

Similarly, we can show that also (20c) holds. Note that, as $W$ is not differentiable with respect to $\kappa$, but as the component in $F(\xi)$ corresponding to $\kappa$ is zero, $\langle\nabla U(\xi), F(\xi)\rangle$ can still be evaluated with a slight abuse of notation. As such, for all $(\tau, \kappa) \in \mathbb{R}_{\geq 0} \times \mathbb{N}$ and almost all $(x, e) \in \mathbb{R}^{m_{x}} \times \mathbb{R}^{m_{e}}$, 
it holds that

$$
\begin{aligned}
\langle\nabla U(\xi), & F(\xi)\rangle \\
= & \langle\nabla V(x), f(x, e)\rangle+\gamma \dot{\phi}(\tau) W^{2}(\kappa, e) \\
& +2 \gamma \phi W(\kappa, e)\left\langle\frac{\partial W(\kappa, e)}{\partial e}, g(x, e)\right\rangle \\
\stackrel{(8),(10)}{\leq}-\varepsilon^{2}|x|^{2}-\underline{\alpha}_{W}^{2} \varepsilon^{2}|e|^{2}-H^{2}(x)+\gamma^{2} W^{2}(\kappa, e) & -\gamma W^{2}\left(2 L \phi(\tau)+\gamma\left(\phi^{2}(\tau)+1\right)\right) \\
& \quad+2 \gamma \phi(\tau) W(\kappa, e)(L W(\kappa, e)+H(x)) \\
= & -\varepsilon^{2}|x|^{2}-\underline{\alpha}_{W}^{2} \varepsilon^{2}|e|^{2}-H^{2}(x) \\
& \quad+2 \gamma \phi(\tau) W(\kappa, e) H(x)-\gamma^{2} W^{2}(\kappa, e) \phi^{2}(\tau) \\
= & -\varepsilon^{2}|x|^{2}-\underline{\alpha}_{W}^{2} \varepsilon^{2}|e|^{2}-(H(x)-\gamma \phi(\tau) W(\kappa, e))^{2} \\
\leq & -\varepsilon^{2}|x|^{2}-\underline{\alpha}_{W}^{2} \varepsilon^{2}|e|^{2} \stackrel{(20 \mathrm{a})}{\leq}-\varepsilon^{2} \min \left\{1, \underline{\alpha}_{W}^{2}\right\} \bar{\alpha}_{U}^{-1} U(\xi)
\end{aligned}
$$

Hence, (20c) is also satisfied with $\lambda_{0}=\varepsilon^{2} \min \left\{1, \underline{\alpha}_{W}^{2}\right\} \bar{\alpha}_{U}^{-1}$.

To complete now the proof, it has to be shown how the "optimal' value of $\mu$ (cf. $\sigma$ ) is obtained while satisfying (23). Note that when we take $\mu$ such that $\mu<e^{\lambda_{0} \tau_{\text {miati }}}$, there indeed exists a $\tilde{\lambda} \in\left(0, \lambda_{0}\right)$ such that (23) holds. Moreover, observe that we obtain the largest value for $\tau_{\text {mati }}$ when $\sigma$ is as small as possible, or, hence, when $\mu$ is as large as possible. As such, using the results above, it follows that we ideally would like to have that $\mu$ is as large as possible with its upper bound given by

$$
\mu<e^{\varepsilon^{2} \min \left\{1, \underline{\alpha}_{W}^{2}\right\}} \frac{1}{\max \left\{\bar{\alpha}_{V}, \gamma \sqrt{\mu} \lambda^{-1} \bar{\alpha}_{W}^{2}\right\}} \tau_{\text {miati }} .
$$

Using now the properties of the Lambert W-function [22], it can be shown that (26) results in

$$
\mu<\min \left\{e^{2 \tilde{\varepsilon} \bar{\alpha}_{V}^{-1} \tau_{\text {miati }}}, e^{2 W_{L}\left(\tilde{\varepsilon} \frac{\lambda}{\gamma \bar{\alpha}_{W}^{2}} \tau_{\text {miati }}\right)}\right\}>1 \text { for } \tau_{\text {miati }}>0,
$$

with $\tilde{\varepsilon}:=\frac{1}{2} \varepsilon^{2} \min \left\{1, \underline{\alpha}_{W}^{2}\right\}$, from which the bound on $\sigma$ as in item 5) of Theorem 1 directly follows as $\sigma=\frac{\lambda}{\sqrt{\mu}}$. This completes the proof.

\section{APPENDIX B}

\section{PROOF OF THEOREM 2}

Consider again the Lyapunov function as given in (24). Following the same steps as in the proof of Theorem 1, one can conclude that (20a) and (20b) hold, and during flow (i.e., when $\left.\tau \in\left[0, \tau_{\text {mati }}\right]\right)$, we obtain for all $(\tau, \kappa) \in \mathbb{R}_{\geq 0} \times \mathbb{N}$ and almost all $(x, e) \in \mathbb{R}^{m_{x}} \times \mathbb{R}^{m_{e}}$ that

$$
\begin{aligned}
&\langle\nabla U(\xi), F(\xi)\rangle \\
&=\langle\nabla V(x), f(x, e)\rangle+\gamma \dot{\phi}(\tau) W^{2}(\kappa, e) \\
&+2 \gamma \phi W(\kappa, e)\left\langle\frac{\partial W(\kappa, e)}{\partial e}, g(x, e)\right\rangle \\
& \leq-\varepsilon^{2} V(x)-H^{2}(x)+\gamma^{2} W^{2}(\kappa, e) \\
&-\gamma W^{2}\left(2 L \phi(\tau)+\gamma\left(\phi^{2}(\tau)+1\right)\right) \\
&-\gamma^{2} W^{2}(\kappa, e) \phi^{2}(\tau)-\varepsilon^{2} \gamma \phi(\tau) W^{2}(\kappa, e) \\
&=-\varepsilon^{2} V(x)-\varepsilon^{2} \gamma \phi(\tau) W^{2}(\kappa, e)-(H(x)-\gamma \phi(\tau) W(\kappa, e))^{2} \\
& \leq-\varepsilon^{2} V(x)-\varepsilon^{2} \gamma \phi(\tau) W^{2}(\kappa, e)=-\varepsilon^{2} U(\xi) .
\end{aligned}
$$

Hence, (20c) is also satisfied with $\lambda_{0}=\varepsilon^{2}$. As such, we obtain in this case that the highest bound on $\tau_{\text {mati }}$ can be obtained when $\mu$ is taken as large as possible with its upper bound given by $\mu<e^{\varepsilon^{2} \tau_{\text {mati }}}$, or, hence, when $\sigma$ is taken as small as possible with $\sigma>\lambda e^{-\frac{1}{2} \varepsilon^{2} \tau_{\text {mati }}}$. This completes the proof.

\section{REFERENCES}

[1] J. P. Hespanha, P. Naghshtabrizi, and Y. Xu, "A survey of recent results in networked control systems," Proc. IEEE, vol. 95, no. 1, pp. 138-162, Jan. 2007.

[2] G. C. Walsh, O. Beldiman, and L. G. Bushnell, "Asymptotic behavior of nonlinear networked control systems," IEEE Trans. Autom. Control, vol. 46, no. 7, pp. 1093-1097, Jul. 2001.

[3] G. C. Walsh, H. Ye, and L. G. Bushnell, "Stability analysis of networked control systems," IEEE Trans. Control Syst. Technol., vol. 10, no. 3, pp. 438-446, May 2002.

[4] D. Nešić and A. R. Teel, "Input-to-state stability of networked control systems," Automatica, vol. 40, no. 12, pp. 2121-2128, 2004

[5] D. Nešić and A. R. Teel, "Input-output stability properties of networked control systems," IEEE Trans. Autom. Control, vol. 49, no. 10, pp. 1650-1667, Oct. 2004.

[6] D. Carnevale, A. R. Teel, and D. Nešić, "A Lyapunov proof of an improved maximum allowable transfer interval for networked control systems," IEEE Trans. Autom. Control, vol. 52, no. 5, pp. 892-897, May 2007.

[7] D. Nešić, A. R. Teel, and D. Carnevale, "Explicit computation of the sampling period in emulation of controllers for nonlinear sampled-data systems," IEEE Trans. Autom. Control, vol. 54, no. 3, pp. 619-624, Mar. 2009.

[8] M. Tabbara, D. Nešić, and A. R. Teel, "Stability of wireless and wireline networked control systems," IEEE Trans. Autom. Control, vol. 52, no. 9 , pp. $1615-1630$, Sep. 2007.

[9] W. P. M. H. Heemels, A. R. Teel, N. van de Wouw, and D. Nešić "Networked control systems with communication constraints: Tradeoffs between transmission intervals, delays, and performance," IEEE Trans. Autom. Control, vol. 55, no. 8, pp. 1781-1796, Aug. 2010

[10] R. Postoyan, N. Van de Wouw, D. Nešić, and W. P. M. H. Heemels "Tracking control for nonlinear networked control systems," IEEE Trans. Autom. Control, vol. 59, no. 6, pp. 1539-1554, Jun. 2014.

[11] S. H. J. Heijmans, D. P. Borgers, and W. P. M. H. Heemels, "Stability and performance analysis of spatially invariant systems with networked communication," IEEE Trans. Autom. Control, to be published, doi: 10.1109/TAC.2017.2676983.

[12] K.-Z. Liu, R. Wang, and G.-P. Liu, "Tradeoffs between transmission intervals and delays for decentralized networked control systems based on a gain assignment approach," IEEE Trans. Circuits Syst. II, Exp. Briefs, vol. 63, no. 5, pp. 498-502, May 2016.

[13] K. Liu, E. Fridman, and L. Hetel, "Stability and $l_{2}$-gain analysis of networked control systems under round-robin scheduling: A time-delay approach," Syst. Control Lett., vol. 61, no. 5, pp. 666-675, 2012.

[14] M. C. F. Donkers, W. P. M. H. Heemels, N. Van de Wouw, and L. Hetel "Stability analysis of networked control systems using a switched linear systems approach," IEEE Trans. Autom. Control, vol. 56, no. 9 pp. 2101-2115, Sep. 2011.

[15] C. Briat, "Convex conditions for robust stability analysis and stabilization of linear aperiodic impulsive and sampled-data systems under dwell-time constraints," Automatica, vol. 49, no. 11, pp. 3449-3457, 2013.

[16] L. Hetel et al., "Recent developments on the stability of systems with aperiodic sampling: An overview," Automatica, vol. 76, pp. 309-335, Feb. 2017.

[17] N. W. Bauer, P. J. H. Maas, and W. P. M. H. Heemels, "Stability analysis of networked control systems: A sum of squares approach," Automatica vol. 48, no. 8, pp. 1514-1524, 2012.

[18] J. P. Hespanha and A. S. Morse, "Stability of switched systems with average dwell-time," in Proc. IEEE 38th Conf. Decis. Control, Phoenix, AZ, USA, 1999, pp. 2655-2660.

[19] J. P. Hespanha, D. Liberzon, and A. R. Teel, "Lyapunov conditions for input-to-state stability of impulsive systems," Automatica, vol. 44 , no. 11, pp. 2735-2744, 2008.

[20] D. Liberzon, Switching in Systems and Control. Boston, MA, USA Birkhaüser, 2003

[21] R. Goebel, R. G. Sanfelice, and A. R. Teel, Hybrid Dynamical Systems. Oxford, U.K.: Princeton Univ. Press, 2012.

[22] R. M. Corless, G. H. Gonnet, D. E. G. Hare, D. J. Jeffrey, and D. E. Knuth, "On the Lambert $W$-function," $A d v$. Comput. Math., vol. 5 no. 1 , pp. $329-359,1996$. 